\title{
Auf dem Weg zu einem kooperativen Menschenbild Überlegungen zur Organisation der Kooperation
}

\author{
MICHÈLE MORNER UND NADINE WÄLDER ${ }^{* *}$
}

Neue Erkenntnisse der Verhaltens- und Neuroökonomie stellen das Menschenbild des Homo oeconomicus zunehmend in Frage und rücken einen kooperativen Akteur ins Zentrum der wirtschaftswissenschaftlichen Diskussion sowie der unternehmerischen Praxis. Auf Grundlage von Elinor Ostroms Forschung zu konditionaler Kooperationsbereitschaft und kollektiver Selbststeuerung in sozio-ökologischen Systemen zeigt der vorliegende Beitrag, wie Unternehmen einen Rahmen schaffen können, der $\mathrm{zu}$ einer entsprechenden Kooperationsbereitschaft beim Individuum führt. Treiber der Kooperation sind dabei Reputation, Vertrauen, Solidarität und sich herauskristallisierende Kooperationsnormen.

Schlagwörter: Kooperation, Menschenbild, Organisation, Vertrauen, Reputation, Solidarität, Kooperationsnormen

\section{Towards a Cooperative Idea of Man in Organizations}

New research in behavioral and neuro-economics has shattered the belief in the basic assumptions behind the homo oeconomicus and has shown the need for a more cooperation-oriented idea of man. Simultaneously, the issue of cooperation has become more and more important in organizational life. We thus show how organizations can establish conditions that encourage individuals to cooperate decentrally. Combining Elinor Ostrom's elaborated research on collective self-governance in socioecological systems with organizational theory and transferring it to companies, we show how reputation, trust, solidarity and norms can be built up as the main drivers for fostering cooperation.

Keywords: Cooperation, Human Actor, Organizing, Trust, Reputation, Solidarity, Cooperation Norms

Beitrag eingereicht am 15.11.2012; nach doppelt verdecktem Gutachterverfahren überarbeite Fassung angenommen am 25.07.2013.

** Univ.-Prof. Dr. Michèle Morner, Deutsche Universität für Verwaltungswissenschaften Speyer, Lehrstuhl für Personal, Führung und Entscheidung im öffentlichen Sektor, Freiherr-vom-SteinStraße 2, D-67346 Speyer, Tel.: +49-(0)6232 654-275, Fax: +49-(0)6232 654-279 und ReinhardMohn-Institut für Unternehmensführung \& Corporate Governance, Alfred-Herrhausen-Straße 50, 58448 D-Witten, Tel.: +49-(0)2302 926-517, Fax: +49-(0)2302 926-587, E-Mail: michele.morner@uni-wh.de, und E-Mail: morner@uni-speyer.de, Forschungsschwerpunkte: Organisation und Management multidivisionaler Unternehmen, Behavioral Corporate Governance.

Nadine Wälder, M.A., Doktorandin am Reinhard-Mohn-Institut für Unternehmensführung \& Corporate Governance, Alfred-Herrhausen-Straße 50, D-58448 Witten, Fax: +49-(0)2302 926587, E-Mail: nadine.waelder@uni-wh.de, Forschungsschwerpunkte: Kooperation und Zusammenarbeit, kollektives Handeln, Organisation und Steuerung von Unternehmen, Steuerung von Wissen. 


\section{Einleitung}

Nichts prägt eine Gesellschaft so sehr, wie ihre Vorstellung vom Menschen. Nichts prägt die Wirtschaftswissenschaft so sehr, wie das Menschenbild des Homo oeconomicus. Auch wenn der allwissende, rein rational und opportunistisch agierende Homo oeconomicus nur eine Ansammlung bestimmter Modellannahmen ist und keine Aussagen darüber macht, wie der Mensch tatsächlich beschaffen ist oder sein sollte, prägt er unsere Wahrnehmung der Wirklichkeit, unsere Einstellungen und damit - gewissermaßen durch die Hintertür - unser Verhalten. In diesem Sinne plädiert die kürzlich verstorbene Nobelpreisträgerin Elinor Ostrom für ein Menschenbild mit anderen, weniger egoistischen Verhaltensannahmen. In ihren Augen sind Menschen durchaus uneigennützig kooperationsbereit, und nur wenn wir diese Kooperationsbereitschaft als Grundannahme in wirtschaftswissenschaftlichen Theorien zulassen, können wir der Realität gerecht werden. Wie das Menschenbild eines derartigen Homo conditional cooperativus aussehen kann, und wie organisatorische Rahmenbedingungen zu schaffen sind, welche die Kooperation im Unternehmen fördern, sind die Fragen, die dieser Beitrag beantworten möchte.

Kooperationsbereitschaft ist auch deswegen in Unternehmen unerlässlich, weil sie zunehmend Herausforderungen gegenüberstehen, die nicht mehr alleine durch hierarchische Steuerung lösbar sind. Im Besonderen lassen sich wissensintensive Prozesse, wie sie häufig in der Forschung und Entwicklung, aber auch bei dienstleistungsorientierten Unternehmen vorkommen, nur sehr schwer durch direkte Weisung, von oben festgelegten Regeln und/oder mit Kennzahlen steuern (vgl. Frey/Osterloh 2002: 23ff.; Frost/Morner 2005: 33; Osterloh/Weibel 2009: 150-153). Es gilt vielmehr, die Individuen direkt an den Entscheidungs- und Handlungsprozessen zu beteiligen und durch Selbststeuerung Kooperation zu fördern (vgl. Frost/Morner 2010: 217).

Die Realität zeigt, dass Individuen auch ohne egoistische Absicht freiwillig kooperieren (vgl. Ostrom 2000: 138; Frey/Meier 2004: 1717). Dies wird von neueren Erkenntnissen der Neuroökonomik bestätigt. Diese weisen darauf hin, dass dem Menschen eine gewisse Kooperationsbereitschaft angeboren ist, die von bestimmten Rahmenbedingungen gefördert, von anderen unterdrückt wird. ${ }^{1}$ Es wird also höchste Zeit, dass wir einerseits Modelle bzw. Konzepte entwickeln, die auf einem grundsätzlich kooperationsbereiten Menschenbild basieren, und andererseits auch über Bedingungen nachdenken, die die Kooperation in Organisationen langfristig fördern. Damit ist statt des Egoisten im Sinne des Homo oeconomicus nicht unbedingt ein Altruist, sondern vielmehr ein Kooperateur unter bestimmten Bedingungen gefragt. Der vorliegende Beitrag übernimmt dieses Menschenbild von Elinor Ostrom und überträgt es aus ihrem Anwendungsfeld der Kommunen und lokalen Institutionen in den betriebswirtschaftlichen Kontext von Unternehmen (Abschnitt 2). Mit Hilfe neuerer organisations- und motivationstheoretischer Überlegungen wird ein Modell der Organisation von Kooperation entwickelt, das organisatorische Bedingungen aufzeigt, unter denen in Unternehmen Kooperation gefördert wird. Darauf aufbauend werden Implikationen für die Förderung der Kooperationsbereitschaft gegeben und es wird in diesem Zusammenhang die bedeutende Rolle von Selbststeuerung aufgezeigt und wie diese in

$1 \quad$ Vgl. zur Vertiefung z.B. Elger (2008) oder Camerer et al. (2005). 
Unternehmen gefördert werden kann (Abschnitt 3). Die Schlussfolgerungen fassen die zentralen Inhalte dieses Beitrags nochmals zusammen (Abschnitt 4).

\section{Der Homo conditional cooperativus als Kooperateur unter bestimmten Bedingungen}

Die Annahmen des Homo oeconomicus, die nach wie vor vielen wirtschaftswissenschaftlichen Theorien zugrunde liegen, erlauben nur eine Kooperation in egoistischer Form. Es handelt sich bei diesem Menschenbild um einen Homo non-cooperativus (Abschnitt 2.1). Auf Basis eines solchen Menschenbildes lässt sich jedoch kaum eruieren, wie auch nicht-egoistische Kooperation in Organisationen gefördert werden kann. Dazu notwendig ist die Entwicklung eines Menschenbildes, das unter bestimmten Bedingungen auch Kooperation erlaubt: der Homo conditional cooperativus (Abschnitt 2.2).

\subsection{Der Homo oeconomicus als Homo non-cooperativus}

Menschenbilder sind Annahmen über das menschliche Verhalten, die die Realität vereinfachen, um darauf basierend entsprechende Modelle und Theorien zu entwickeln (vgl. z.B. König/Volmer 2005: 34). Das maßgebliche Konzept in den Wirtschaftswissenschaften basiert dabei auf der Annahme von rational getroffenen, an eigenen Präferenzen ausgerichteten Entscheidungen. Diese beschreibt bereits Leon Walras (1954/1874) als Grundlage allen menschlichen Handelns. Etwas später verwendet Vilfredo Pareto (1969/1906) erstmals den lateinischen Begriff des Homo oeconomicus für den Menschen mit entsprechend individuellem rationalem Nutzenkalkül. Dabei liegen dem ursprünglichen Konzept des Homo oeconomicus mit der Annahme vollkommener Rationalität (Abschnitt 2.1.1) und der Annahme strikten Eigennutzes (Abschnitt 2.1.2) zwei Extremannahmen zugrunde. Für die Argumentation im vorliegenden Beitrag relevant ist vor allem die Annahme des strikten Eigennutzes. Sowohl Rationalität- als auch Eigennutzannahme wurden inzwischen mehrfach relativiert, erscheinen aber - auch in ihrer relativierten Form - als Grundlage für die Modellbildung in den Wirtschaftswissenschaften nicht unproblematisch (Abschnitt 2.1.3).

\subsubsection{Die Annahme vollkommener Rationalität}

In seinem ursprünglichen Verständnis handelt der Homo oeconomicus nicht nur rational, sondern kennt alle ihm zur Verfügung stehenden Alternativen bzw. deren Konsequenzen; er ist allwissend. Schon von Neumann und Morgenstern (1947) haben jedoch gezeigt, dass für den Homo oeconomicus nicht unbedingt alle Konsequenzen einer Handlungsalternative bekannt sein müssen. Es würde reichen, wenn er die möglichen Resultate der Alternativen und deren Eintrittswahrscheinlichkeiten kennt. Noch weitergehender führte Herbert Simon in der Organisationsforschung das Konzept der begrenzten Rationalität ein und betont damit unter anderem, dass der Nutzen der Akteure nicht unbedingt maximiert werden muss, sondern auch satisfiziert werden kann (vgl. Simon 1955: 101; Williamson 1985: 44). Der Realität entspricht jedoch auch die so relativierte Rationalitätsannahme nicht. So zeigen Daniel Kahnemann und Amos Tversky (1979) in ihrer Prospect Theory, dass der Mensch seine Optionen über- 
haupt nur selten rational bewertet; er handelt eher nach einfachen Daumenregeln und überschätzt sich dabei regelmäßig. In den Mittelpunkt rücken plötzlich Phänomene, die klassische Ökonomen nur als Anomalien abtun konnten. Diese Ergebnisse wurden inzwischen in mehreren Studien der Verhaltensökonomik bestätigt (vgl. Tversky/Kahneman 1986; Kahneman et al. 1991; Kahneman 2003).

Doch nicht nur die Ergebnisse der Verhaltensökonomik stellen die Annahme vollkommener Rationalität in Frage, sondern auch die der Neuroökonomik. ${ }^{2}$ Diese verweist darauf, dass in vermeintlich rationalen Entscheidungssituationen Hirnzentren aktiv sind, die eigentlich für unbewusste Aktivitäten zuständig sind. Dazu gehört unter anderem der für die Kooperationsbereitschaft zuständige Bereich, der grundsätzlich in jedem Gehirn des Homo sapiens vorhanden ist und unter bestimmten Bedingungen aktiviert wird.

\subsubsection{Die Annahme strikten Eigennutzes}

In seiner strikten, ursprünglichen Form bedeutet die Annahme des Eigennutzes, dass der Homo oeconomicus sich nur an seinen Präferenzen orientiert und seinen Nutzen rein egoistisch maximiert. Diese Annahme findet sich bereits bei Adam Smith (1999/1776: 17) und wurde später von der Volks- in die Betriebswirtschaftslehre transferiert. Entsprechend bildet das opportunistisch handelnde Individuum auch die Basis institutionenökonomischer Organisationstheorien. Der Opportunismus der Akteure wird vor allem durch die Kombination mit der - bereits unter Abschnitt 2.1.1 beschriebenen - begrenzten Rationalität (vgl. Williamson 1985: 44) zum Problem. Somit wird der Opportunismus des Einzelnen nämlich durch unvollständig zur Verfügung stehende Informationen bzw. eine verzögerte Bereitstellung dieser (vgl. Kirchgässner 2008: 67f.) gefördert und mündet unter Umständen in "Betrug, Lug und Täuschung" (vgl. Williamson 1985: 30, 47ff.).

Vertreter der experimentellen Ökonomik haben gezeigt, dass die Annahme des strikten Eigennutzes nicht der Realität entspricht. Menschen verhalten sich nicht immer egoistisch, sondern unter bestimmten Bedingungen durchaus kooperativ und fair. Selbst wenn diese Erkenntnis nicht unbedingt zu einer Ablehnung des Konzeptes des Homo oeconomicus führen muss, erweitert sie dessen Nutzenfunktion um eine Präferenz für soziale Gleichverteilung. Hier stellt sich freilich die Frage, wie weit man die Eigennutzannahme „dehnen“ kann, so dass noch vom Homo oeconomicus die Rede ist. Nicht mehr subsumierbar ist der Fall der „unegoistischen“ Kooperation. Dass diese in der Realität durchaus vorkommt, zeigen wiederum Studien der Neuroökonomik. ${ }^{3}$ Demzufolge sorgen bestimmte Gehirnbereiche für normatives Verhalten und ein entsprechendes Fairnessempfinden (vgl. Spitzer et al. 2007: 192f.). ${ }^{4}$

\footnotetext{
2 Vgl. zur Vertiefung z.B. Elger (2008) oder Camerer et al. (2005).

3 Vgl. zur Vertiefung z.B. Elger (2008) oder Camerer et al. (2005).

4 Spitzer et al. verweisen dabei auf den rechten dorsolateralen präfrontalen Kortex, welcher entscheidend an fairnessorientiertem, normativen Verhalten beteiligt ist (vgl. Spitzer et al. 2007: 19f.).
} 


\subsubsection{Beeinflussung der Realität}

Die Realitätsferne der Annahmen des Homo oeconomicus stellt zunächst kein Problem dar. Ziel eines - wie auch immer gewählten - Menschenbildes ist ja eben gerade nicht die identische Abbildung der komplexen Welt, sondern deren Vereinfachung. Und das Konzept des Homo oeconomicus will ja auch gar keine Aussagen darüber machen, wie der Mensch tatsächlich beschaffen ist oder sein sollte. Dennoch zeigen empirische Ergebnisse, dass die mit einem Menschenbild verbundenen Modellannahmen einen zumindest indirekten Einfluss auf die Wirklichkeit haben. Sie beeinflussen nämlich die Wahrnehmung derer, die mit diesen Modellen arbeiten, und daran anschließend auch deren Verhalten. Erläutert man Akteuren beispielsweise eine spezifische Situation aus der Perspektive des Modells, ändern sie ihr tatsächliches Verhalten und handeln in erhöhtem Ausmaß dem Modell entsprechend (vgl. Morgenstern 1972: 706). Das so veränderte Verhalten wird außerdem auch auf ganz andere Zusammenhänge übertragen. So zeigen beispielsweise Experimente, dass Studenten der Wirtschaftswissenschaften am Ende ihrer Ausbildung deutlich eigennützigere Verhaltensweisen an den Tag legen als ihre Kommilitonen anderer Fachrichtungen (vgl. Enste et al. 2009: 60f.; Frey et al. 2010: 318ff.; Adler 2002: 149). ${ }^{5}$ Unter Umständen führt damit ein zu einseitiger Fokus auf den Homo oeconomicus in der Managerausbildung dazu, dass Führungskräfte ihre Mitarbeiter tatsächlich als Nutzenmaximierer und Opportunisten sehen und so behandeln. Im Sinne der sich selbst-erfüllenden Prophezeiung provoziert eine derartige Form der Führung dann tatsächlich ein egoistisches und opportunistisches Verhalten von Geführten, wie bereits McGregor aufgezeigt hat (vgl. McGregor 1966: 7ff.).

\subsection{Der Mensch als Homo conditional cooperativus und die Rolle von Nor- men}

Die Praxis zeigt, dass Individuen häufiger kooperieren als von bestehenden Theorien ${ }^{6}$ angenommen. So findet Elinor Ostrom beispielsweise in den von ihr untersuchten sozio-ökologischen Systemen von vier verschiedenen Typen von Menschen nur einen, der rein egoistisch agiert (vgl. Ostrom 2000: 142). Alle anderen drei kooperieren selbst wenn es ihrer Nutzenmaximierung widerspricht: Der rein altruistische Typus kooperiert völlig unegoistisch und losgelöst von seiner Nutzenmaximierung. Daneben gibt es noch den sich moralisch verhaltenden, normenkonformen Typus, der entsprechend von ihm anerkannter Normen kooperiert. Dieser, von Elinor Ostrom bezeichnete konditional Kooperative, kooperiert nur unter bestimmten Bedingungen (vgl. Ostrom 2000: 141ff.). Als Erweiterungstypus des normkonformen Akteurs identifiziert Elinor Ostrom den so genannten „willing punisher“, welcher sich für die Einhaltung der von ihm anerkannten Normen einsetzt. Er ist gewillt, all diejenigen zu bestrafen, die nicht normenkonform handeln und als Trittbrettfahrer auftreten. Genauso

Die Studien belegen weiterhin, dass vier von fünf Ökonomen die neoklassische Theorie der Ökonomie als bedeutsamste erachten. Zudem wird der Homo oeconomius von zwei Dritteln der befragten Ökonomen als durchaus brauchbares Menschenbild angesehen, das keineswegs verzerrte menschliche Verhaltensmuster der Realität aufzeigt (vgl. Enste et al. 2009: 60f.; Frey et al. 2010: 318ff.).

6 Hier seien die neoklassische Theorie und die Institutionenökonomie als Beispiele zu nennen. 
belohnt er jedoch diejenigen, die sich normkonform verhalten. Im Besonderen die Anwesenheit der letzten beiden Typen, des konditional Kooperativen und in seiner Erweiterung des „willing punishers“, trägt dazu bei, eine Kooperationsatmosphäre zu schaffen und eine bestehende Kooperation zu intensivieren (vgl. Ostrom 2000: 141ff.). Die drei kooperationswilligen Typen sind nicht nur in den Augen Ostroms in der Überzahl: Studien belegen, dass 60 Prozent aller Menschen in der Regel zumindest dann kooperieren, wenn sie um die Kooperation anderer wissen (vgl. Fischbacher et al. 2001: 397ff.; Frey/Meier 2004: 1718ff.). Sie senken ihre Kooperationsbereitschaft erst, wenn die jeweils anderen auf Dauer nicht kooperieren und entsprechende Kooperationsnormen fehlen (vgl. Frey/Meier 2004: 1721).

\section{Auf dem Weg zu einem theoretischen Bezugsrahmen der Organisation von Kooperation}

Gemäß organisationstheoretischer Überlegungen setzt die Entstehung von Kooperationsbereitschaft den Aufbau von Reputation, Vertrauen und Solidarität bei den Beteiligten voraus (siehe für einen Überblick Abbildung 1). Man spricht in diesem Zusammenhang auch von so genannten „weichen“ Integrationsmechanismen, die den Grundstein für eine freiwillige Zusammenarbeit in Organisationen legen (Abschnitt 3.1). Gelingt es durch Reputation, Vertrauen und Solidarität, die Kooperationsbereitschaft zu erhöhen, steigen bei daraus folgender tatsächlich stattfindender Kooperation wiederum Reputation, Vertrauen und Solidarität der beteiligten Akteure. Es entsteht ein sich selbst verstärkender Prozess als funktionierender Kooperationszyklus, der wiederum dazu führt, dass sich entsprechende Kooperationsnormen herauskristallisieren (Abschnitt 3.2). Die Kooperationsnormen sind jedoch nicht nur Ergebnis dieses Prozesses, sondern auch vice versa wichtige Grundlage davon (Abschnitt 3.3). Von besonderer Bedeutung für die Entstehung von Kooperationsnormen und damit für den funktionierenden Kooperationsprozess sind spezifische Kooperationspraktiken, die einen geeigneten Rahmen für die Etablierung von Kooperationsnormen bilden. Elinor Ostrom bezeichnet sie als „Best Practices“ der Kooperation, die - wie sie am Beispiel der von ihr untersuchten sozio-ökologischen Systeme aufzeigt - allen langfristig gut funktionierenden Kooperationsprozessen gemein sind (Abschnitt 3.4).

\subsection{Die Rolle von Reputation, Vertrauen und Solidarität bei der Entstehung von Kooperationsbereitschaft}

Im Mittelpunkt eines funktionierenden Kooperationsprozesses steht die Kooperationsbereitschaft der Beteiligten. Deren Entstehung basiert vor allem auf dem Vorhandensein der so genannten ,weichen“ Integrationsmechanismen Reputation, Vertrauen und Solidarität (siehe nochmals Abbildung 1). Während dieser Zusammenhang bei Ostrom nur ansatzweise und mit besonderem Fokus auf das Vertrauen skizziert wird, reichern wir ihre Überlegungen mit Erkenntnissen aus der Organisationstheorie an und zeigen, wie Reputation (Abschnitt 3.1.1), Vertrauen (Abschnitt 3.1.2) und Solidarität (Abschnitt 3.1.3) positiv auf die Kooperationsbereitschaft in Unternehmen wirken und vice versa die tatsächlich stattfindende Kooperation das Vertrauen in die Kooperierenden festigt, deren Reputation erhöht und die gemeinsame Solidarität ent- 
faltet. Ein sich selbst verstärkender Kreislauf entsteht und gleichzeitig bilden und verstärken sich entsprechende Kooperationsnormen.

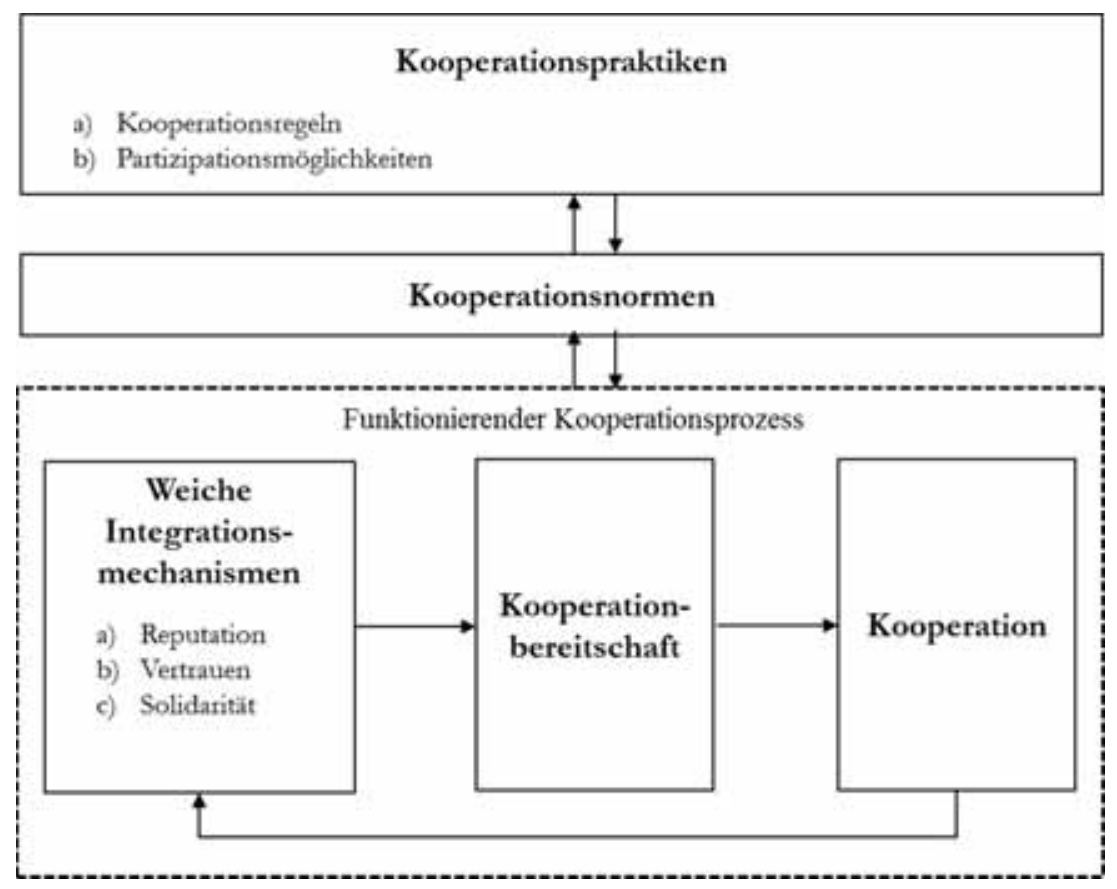

Abbildung 1: Bezugsrabmen der Organisation von Kooperation (Quelle: eigene Darstellung)

\subsubsection{Reputation, Kooperationsbereitschaft und Kooperation}

Als Reputation werden Eigenschaften und Verhaltensmuster, aber auch Fähigkeiten und Erfahrungen bezeichnet, die einer Person zugesprochen werden (vgl. Ringlstetter 1997: 158; Kilduff/Krackhardt 1994: 89). Im Zusammenhang mit der Kooperationsbereitschaft ist dabei insbesondere das bisherige Kooperationsverhalten einer Person maßgeblich. Im Hinblick auf die Kooperation können so vertrauenswürdige von vertrauensunwürdigen Personen unterschieden und mögliche Kooperationspartner identifiziert werden, mit welchen das Risiko, bei einer Zusammenarbeit enttäuscht zu werden, sinkt (vgl. Granovetter 1985: 487-493). Die Reputation einer Person erhöht ihre Akzeptanz bei potentiellen Kooperationspartnern und fördert so deren Kooperationsbereitschaft (vgl. Fehr/Gächter 2002: 137-140). Rückkoppelnd wirkt sich die tatsächliche Kooperation, in der Akteure positiv als Kooperationspartner wahrgenommen werden, positiv auf die Reputation der Beteiligten aus. Sie ist gleichbedeutend mit dem Qualitätssiegel, ein vertrauenswürdiger Kooperationspartner zu sein (vgl. Granovetter 1985: 487-493; Ostrom 1998: 12). 


\subsubsection{Vertrauen, Kooperationsbereitschaft und Kooperation}

Das Gegenstück zu Reputation ist Vertrauen (vgl. Ostrom 1998: 12). Es drückt die Erwartung in die Vertrauenswürdigkeit des jeweils anderen aus (vgl. Burt/Knez 1995: 70) und wird so als antizipierte Kooperation verstanden. Damit reduziert Vertrauen die Unsicherheit darüber, ob der andere kooperiert (vgl. Ostrom 1998: 12; Kramer 1999: 571 $)^{7}$ und nimmt so eine zentrale Rolle dabei ein, Kooperationsbereitschaft zu erhalten und zu schaffen. Bei positiven Kooperationserfahrungen kann im Laufe der Zeit das Vertrauen in ein Individuum wachsen (vgl. Kramer 1999: 575f.; Ringlstetter 1997: 159). Dies gilt natürlich nur so lange, wie das entgegengebrachte Vertrauen nicht missbraucht wird (vgl. Ostrom 2010b: 660f.). Der positive Kooperationsausgang intensiviert im Gegenzug wiederum das Vertrauen in die Kooperationspartner (vgl. Ostrom 1998: 12f.; Kramer 1999: 575f.).

\subsubsection{Solidarität, Kooperationsbereitschaft und Kooperation}

Das Konzept der Solidarität stellt eine Erweiterung des Vertrauens dar, indem diese zusätzlich die Interessen der jeweils anderen berücksichtigt (vgl. Ringlstetter 1997: 160). Sie basiert also nicht nur auf (tatsächlichen oder antizipierten) Kooperationserfahrungen, sondern berücksichtigt die Ziele anderer Mitarbeiter, aber auch übergreifende Ziele, z.B. des Unternehmens oder eines spezifischen Unternehmensteiles. Dass Mitarbeiter ihr Handeln in Unternehmen daran orientieren und in diesem Sinne durch Solidarität zum Unternehmen und ihren Kollegen geleitet werden, zeigt bereits Elton Mayo (2003/1933). So werden Gruppenziele als eigene Ziele aufgefasst (vgl. de Cremer/van Dijk 2002: 321ff.). Darüber hinausgehend lässt sich Solidarität als positive Wahrnehmung einer bestimmten Gruppe und ihren Mitgliedern definieren und resultiert in einem hohen Grad an Gruppenidentifikation, wobei aus einer „Ich“-Sichtweise ein „Wir“-Gefühl beim Individuum entsteht (vgl. Willer et al. 2012: 125f.). Für die Kooperationsbereitschaft bedeutet dies, dass Solidarität ein Bewusstsein unter den Beteiligten entfachen kann, dass die Kooperation positiv anerkannt wird und somit kooperationshinderliche Aktionen (wie zum Beispiel Handlungen durch Trittbrettfahrer) verhindert werden (vgl. Willer et al. 2012: 127). Tatsächlich stattfindende Kooperation identifiziert solidarische Kooperationspartner und verstärkt unter diesen dementsprechend die bestehende Solidarität durch die positiven Kooperationserfahrungen.

\subsection{Die Rolle von Kooperationsnormen für funktionierende Kooperation}

Durch einen langfristig angelegten Kooperationsprozess können die Akteure ihr Vertrauen in die Kooperationsbereitschaft der anderen festigen, gleichzeitig ihre Reputation erhöhen und die Solidarität steigern. Dies geschieht durch positive Kooperationserfahrungen und das gleichzeitige Erlernen von Kooperationsnormen. Kooperationsnormen helfen den Akteuren, Situationen zu bewerten und auf diese Weise ein gemeinsames Verständnis über Verbot, Erlaubnis und generelle Handlungsmöglichkeiten zu entwickeln (vgl. Posner/Rasmusen 1999: 369f.; Ostrom 1998: 9). Damit erleichtern sie den Aufbau von Reputation, Vertrauen und Solidarität. Wesentliche Grundlage dieser in Kooperationssituationen entstehenden Kooperationsnormen sind

Vgl. zur Vertiefung Luhmann (1994) und Garfinkel (1963). 
die dem Menschen innewohnenden Fairness- (Abschnitt 3.2.1) und Reziprozitätsnormen (Abschnitt 3.2.2) (vgl. Fehr/Gächter 2000: 159; Fehr/Schmidt 2001: 617). Diese sind, zumindest in westlichen Industrienationen (vgl. Franzen/Pointner 2008: 2113f.), die Basis der sozialen Interaktion des Menschen (vgl. Fehr/Schmidt 2001: 617).

\subsubsection{Fairnessnormen als Grundlage für Kooperationsnormen}

Fairnessnormen beziehen sich auf die gleichmäßige Verteilung von Einsatz und Nutzen (vgl. Frey/Benz 2007: 14f.). Für deren Aufbau und daraus resultierend dem Aufbau von Reputation, Vertrauen und Solidarität, kommt es nicht darauf an, ob Prozesse tatsächlich fair ablaufen, sondern darauf, ob sie als fair wahrgenommen werden. Die wahrgenommene Fairness des Individuums spiegelt dabei das Fairnessempfinden des Einzelnen wider und bezieht auch die wahrgenommene positive bzw. negative Absicht der anderen Akteure mit ein (vgl. Falk et al. 2003: 20ff.). Das bedeutet im Fall von Solidarität auch, die Interessen der anderen zu berücksichtigen (vgl. Ostrom 2005: 262f.). Die wahrgenommene Fairness in der Kooperationssituation veranlasst das Individuum wiederum, sich kooperativ zu verhalten oder nicht (vgl. Fehr/Gächter 2000: 172). Experimente belegen, dass der Mensch generell dazu neigt, faires Handeln zu belohnen und unfaires Verhalten zu bestrafen (vgl. Falk et al. 2003: 20; Cox et al. 2007: 18; Frey/Benz 2007: 14f.). Auf die Situation einer Kooperation bezogen, streben Menschen generell eine gleiche Verteilung des Einsatzes und des Nutzens aus der Kooperationssituation an. Diese wird als fair empfunden (vgl. Frey/Benz 2007: 14f.).

\subsubsection{Reziprozitätsnormen als Grundlage der Kooperationsnormen}

Die Norm der Reziprozität liegt dem auf den Fairnessnormen beruhenden, gegenseitigen Geben und Nehmen, bzw. dem „Wie du mir, so ich dir“-Spiel, in Kooperationssituationen zugrunde (vgl. Gouldner 1960: 161; Frey/Benz 2007: 14f.). Dabei ergibt sich der eigene Kooperationswille aus der Kooperationsbereitschaft anderer (vgl. Gouldner 1960: 169). Eine funktionierende Reziprozitätsnorm befähigt die Akteure dazu, ohne die Angst eines Vertrauensmissbrauchs an der Kooperation teilzunehmen. Faires Verhalten gilt als Prämisse für dieses Vertrauensverhältnis (vgl. Fahr/Irlenbusch 2000: 275). Als Folge wird positives Verhalten unter den Akteuren begünstigt und ein Vertrauensmissbrauch, also negatives Verhalten, vermindert (vgl. Keysar et al. 2008: 1280). ${ }^{8}$ Die Reziprozität beeinflusst auf diese Weise die Entscheidung zur Beteiligung an der Kooperation. Positives reziprokes Verhalten ist dementsprechend ein bedeutendes Ergebnis von fairness-orientiertem Verhalten (vgl. Falk et al. 2003: 20), um Kooperationsnormen zu festigen (vgl. Ostrom 2000: 146).

Reziprozitätsnormen tragen dazu bei, Stabilität innerhalb der Kooperation zu generieren, indem sie die Etablierung und Durchsetzung von Regeln eines fairen Miteinanders fördern. Ostrom spricht in diesem Zusammenhang von Kooperationspraktiken, die im nachstehenden Kapitel dargestellt werden. Sie begünstigen wiederum die Entstehung von Kooperationsnormen und werden gleichzeitig, wie von Gouldner (1960) beschrieben, durch die bestehenden Normen gestärkt. Vgl. auch bspw. die Studien von Axelrod und Hamilton (1981), Wedekind (1998) sowie Nowak (2006). 


\subsection{Kooperationspraktiken zur Verstärkung des Kooperationsprozesses}

Reziprozitäts- und Fairnessnormen als wesentliche Voraussetzung für den Kooperationsprozess entfalten sich nicht unter allen Bedingungen, sondern nur unter bestimmten Voraussetzungen. Auch hier lernen wir von Elinor Ostrom (vgl. Ostrom 2000: 143-146). Ihre Forschung zeigt, dass Kooperationsnormen nur unter bestimmten Bedingungen entstehen, die eng mit so genannten Kooperationspraktiken zusammenhängen. Allen erfolgreichen und langfristig überlebenden Systemen sind nämlich bestimmte „Kooperationspraktiken“ gemein, die auf Selbstabstimmung unter den Beteiligten basieren (vgl. Ostrom 2000: 149; Ostrom 1990: 88-102, 185-186; Ostrom 2005: 258-271; Ostrom 2010b: 652f.). Dazu gehören insbesondere auf Selbststeuerung basierende Kooperationsregeln (Abschnitt 3.3.1) und die Partizipation an der Regelerstellung und -überwachung (Abschnitt 3.3.2). Im Folgenden stellen wir diese dar und erläutern, was das für die Entstehung der Kooperationsnormen und die Erhöhung der Kooperationsbereitschaft in Unternehmen bedeutet.

\subsubsection{Kooperationsregeln bei Konflikten, zur Fairness und für Sanktionen}

Kooperationsregeln sind implizite und explizite Regeln über die Art und Weise, wie Kooperation funktionieren soll und wie sich die Individuen in bestimmten Situationen verhalten sollen (vgl. Ostrom 1998: 9f.). Sie beinhalten Konfliktlösungsmechanismen (Punkt a), Regeln zur Fairness der Prozesse (Punkt b) und Möglichkeiten zu graduellen Sanktionen (Punkt c):

(a) Konfliktlösungsmechanismen: Ob Konflikte in Unternehmen zu konstruktiven oder destruktiven Situationen zwischen den Beteiligten führen, hängt von vorhandenen Konfliktlösungsmechanismen ab (vgl. Deutsch 1969, 1975). Konfliktlösungsmechanismen umfassen Anleitungen dazu, wie sich eventuell auftauchende Auseinandersetzungen zwischen den Akteuren mit relativ geringem Aufwand möglichst konstruktiv handhaben lassen (vgl. Ostrom 2005: 267f.). Verlaufen Konflikte konstruktiv und somit positiv, führen sie in der Konsequenz zur Verstärkung der Kooperationsnormen und fördern so den Aufbau von Reputation, Vertrauen und Solidarität, also zu einer verbesserten Beziehung und vermehrter Kooperationsbereitschaft. Ein kooperatives Konfliktmanagement ist dementsprechend für Organisationen wichtig (vgl. Tjosvold 2008: 19-22). Andersherum können bereits etablierte Kooperationsnormen eine Konfliktreduktion herbeiführen, da durch sie ähnliche Vorstellungen zum Kooperationsverhalten vorliegen (vgl. Tsai/Ghoshal 1998: 467f.).

(b) Regeln zu fairen Prozessen: Auch Regeln zu fairen Prozessen sind eine wichtige Voraussetzung für die Etablierung von Kooperationsnormen. Nur so entsteht die Sicherheit für die Akteure, dass sie sich auf die anderen an der Kooperation Beteiligten verlassen können (vgl. Falk et al. 2003: 20). Für Unternehmen ist es dementsprechend bedeutsam, Informationen über die Regeln zu fairen Prozessen bereitzustellen, gleichzeitig diese aber auch an die individuellen Wahrnehmungen der Akteure anzupassen. Dieser Vorgang des „Fairness Monitoring“ empfiehlt für dezentrale Prozesse, welche durch einen hohen Grad an persönlichem Austausch, gemeinsamen Grundeinstellungen und durch eine gegenseitige Vertrauensbasis gekennzeichnet sind, dass gerade Regeln in Form von Verhaltenskodizes und formellen Standards zu einem fairen Empfinden des Prozesses beitragen (vgl. Long et al. 2011: 1047ff.). 
(c) Graduelle Sanktionen: Ein Umfeld wird nur als fair wahrgenommen, wenn auch entsprechende Sanktionsmöglichkeiten existieren, um die Fairness zu erhalten (vgl. Ostrom 2000: 151). Damit wird den Akteuren ein Mechanismus zur Hand gegeben, unfaire Handlungen, wie beispielsweise die Missachtung von Regeln, zu „bestrafen“ (vgl. Poteete et al. 2010: 230; Ostrom 2000: 151). ${ }^{9}$ Das kommt der menschlichen Neigung entgegen, unfaire Handlungen zu sanktionieren und faire Handlungen zu belohnen (siehe Abschnitt 3.2) (vgl. Falk et al. 2003: 20). In langfristig erfolgreichen Kooperationen muss es also Sanktionen für die Missachtung von Kooperationsregeln geben. Idealerweise sind die Sanktionsmöglichkeiten dabei graduell variierbar und können bis zum Ausschluss aus der Gruppe führen (vgl. Ostrom 2005: 266f.). Dadurch verhindern sie Regelverletzungen und unterstützen einen fairen Selbststeuerungsprozess. Dementsprechend wird die Vertrauensbasis gestärkt, welche für den Aufbau von Normen bedeutend ist (vgl. Ringlstetter 1997: 159). Die Sanktionierung des Fehlverhaltens stärkt weiterhin das „Wir“-Gefühl der Kooperationspartner und somit die Solidarität (vgl. Ostrom 1998: 12ff.). Das heißt im Umkehrschluss für Unternehmen, einen sogenannten „Distributions-Effekt“ über eine effiziente Wirkung von Sanktionen herzustellen und somit eventuell auftretende negative Effekte zu reduzieren (vgl. Osterloh/Frey 1997: 309ff.). Ebenfalls sind hierzu die in Punkt (b) dargestellten Verhaltenskodizes für Fehlverhalten entscheidend (vgl. Long et al. 2011: 1047ff.). Wichtig ist dabei, dass die Sanktion auf einer formellen Information beruht und somit einsehbar ist - und nicht lediglich implizit existiert (vgl. Osterloh/Frey 1997: 315ff.).

\subsubsection{Partizipationsmöglichkeiten für die an der Kooperation Beteiligten}

Bereits Vroom (1964) sowie Coch und French (1948) haben gezeigt, dass die Partizipation an Entscheidungsprozessen motivierend wirkt. Aber auch neuere Studien bestätigen, dass die Beteiligung an der Gestaltung und Überwachung der eigenen Aufgaben (vgl. Strauss 1998: 15) zu einer erhöhten Identifikation mit sich daraus entwickelnden Kooperationsnormen führt (vgl. Forde et al. 2006: 298). Die Partizipationsmöglichkeiten, die für langfristige Kooperation wichtig sind, liegen nach Ostrom (1990, 2010a, 2010b) vor allem in der gemeinsamen Erstellung und Gestaltung von Kooperationsregeln (Punkt a), in der Beteiligung bei der Auswahl von Kontroll- und Überwachungsmechanismen (Punkt b) und in einer klaren Definition der Kooperationsgrenzen (Punkt c):

(a) Gemeinsame Erstellung und Gestaltung von Kooperationsregeln: Die gemeinsame Erstellung sowie Gestaltung der Kooperationsregeln ist ein wichtiger Bestandteil der Partizipation als Grundlage funktionierender Kooperation. Dazu müssen alle involvierten Individuen zumindest über einen gewählten Vertreter, welcher im Sinne der Gruppe agiert, an der gemeinschaftlichen Schaffung von Regeln des Miteinanders beteiligt sein. Durch die gemeinsame Entwicklung der Regeln tritt ein gemeinsames Verständnis zu Tage, wie die Kooperation stattfinden soll, was im Folgeschluss zu Reputation, Vertrauen und Solidarität führt (vgl. Ostrom 1998: 9f.). Dadurch werden lokale Kooperationsnormen berücksichtigt und vertieft (vgl. Ostrom 2005: 263ff.). Um eine

9 Elinor Ostrom verwendet in diesem Zusammenhang den Begriff des „willing punishers“ (siehe Abschnitt 2.2), welcher als konditional kooperatives Individuum nicht-kooperatives Verhalten und Handeln innerhalb des Kooperationsprozesses bestraft (vgl. Ostrom 2000: 142f.). 
Partizipation des Akteurs bei der Erstellung und Gestaltung der Kooperationsregeln im Unternehmen zu unterstützen, können zum Beispiel ein Vorschlagswesen, aber auch direkte Feedbackrunden helfen (vgl. Toralla et al. 2012: 2707; Mohr/Zoghi 2008: 275). Die damit verbundene Beteiligung bei der Erstellung und Gestaltung der Kooperationsregeln führt beim Mitarbeiter in Unternehmen zu einer positiven Wahrnehmung seiner Partizipationsmöglichkeiten und verstärkt damit wiederum seine Kooperationsbereitschaft (vgl. Rafaeli 1985: 609).

(b) Auswabl der Kontroll-bzw. Überwachungsmechanismen: Einhergehend mit der Gestaltung der Kooperationsregeln wird idealerweise auch gemeinsam entschieden, wie die Einhaltung der aufgestellten Regeln überwacht werden kann. Dadurch entsteht Vertrauen unter den Beteiligten, dass kein opportunistisches Verhalten den Prozess zerstört (vgl. Ostrom 2005: 265f.). Dies stärkt die Fairnesserwartung der Akteure. Damit einhergehend verstärkt sich das Vertrauen, dass unfaire Handlungen, wie beispielsweise die Missachtung von Regeln, bestraft und positive Handlungen belohnt werden (vgl. Poteete et al. 2010: 230; Ostrom 2000: 151). Auch die Auswahl der Kontroll- bzw. Überwachungsmechanismen, kann ebenso wie in (a) geschildert, durch unternehmerische Prozesse erfolgen, welche die Partizipation des Akteurs wahrscheinlicher machen. Gerade ein funktionierendes Vorschlagswesen und direkte Feedbackrunden sind dabei wichtige Instrumente für ein Unternehmen, um die Mitarbeiter bei der Auswahl der Kontroll- und Überwachungsmechanismen zu beteiligen (vgl. Toralla et al. 2012: 2707; Mohr/Zoghi 2008: 275). Beispielsweise sind aber auch die aus dem Konferenzmanagement bekannten „Open Space“-Methoden (vgl. Owen 2008: 8f.) oder die ebenfalls im Gesundheitsmanagement eingesetzten Fokusgruppen (vgl. Meifert/Kesting 2004: 9) immer anerkanntere Möglichkeiten, Einfluss und Mitwirkungsmöglichkeiten der beteiligten Mitarbeiter zu erhöhen

(c) Klare Definition von Grenzen: Langfristig erfolgreiche Kooperation setzt voraus, dass klar festgelegt ist, wer an der Kooperation teilnimmt und wer nicht (vgl. Ostrom 2005: 260f.). Mit der Mitgliedschaft sind in der Gruppe anerkannte Rechte und Pflichten verbunden (vgl. Seabright 1993: 113f.). Der Einsatz von Sanktionen, aber auch die durch die Kooperation entstehenden Vorteile, sind folglich an die klare Definition von Grenzen gebunden. Damit einher geht auch die Identifikation potentieller vertrauenswürdiger Kooperationspartner. Das Verhalten in vergangenen Situationen als auch die jeweilige Reputation der Beteiligten ist demzufolge entscheidend und verweist auf die wichtige Rolle der langfristig angelegten Zusammenarbeit (vgl. Ostrom 2010a: 157; Poteete et al. 2010: 230; Gouldner/Gouldner 1963: 67ff.). ${ }^{10}$ Dies fördert nicht nur die Reputation des Einzelnen, sondern intensiviert auch die Vertrauensbasis und damit die Bereitschaft mit dieser Person zu kooperieren (vgl. Ostrom 2010a: 158f.). Kooperationsförderlich ist dabei oftmals die Möglichkeit, den eigenen Kooperationsbeitrag identifizieren zu können. Die Identifikation des eigenen Kooperationsbeitrages und des Beitrages der anderen zur Kooperation ermöglicht dem Akteur Sicherheit darüber zu erhalten, dass andere ihren Beitrag zur Kooperation leisten. Nicht

Ostrom macht dies an einem MPCR-Wert (margina per capita return) fest. Ist dieser hoch, werden positive Kooperationserfahrungen mit dem Individuum in Verbindung gebracht und die Kooperation wird wahrscheinlicher (vgl. Poteete et al. 2010: 229ff.). 
nur, dass dadurch die Anzahl der Trittbrettfahrer verringert wird, dies schafft wiederum Vertrauen (vgl. Ostrom 2010a: 159).

\section{Schlussbetrachtung}

Unternehmen sind auf die Kooperation ihrer Mitarbeiter angewiesen. Das gilt insbesondere dort, wo Wissen geteilt und gemeinsam entwickelt werden soll. Entsprechend stellte dieser Beitrag eingangs die Frage, wie sich die Kooperationsbereitschaft der Mitarbeiter fördern lässt. Eine wirtschaftswissenschaftliche Forschung, die diese Frage beantworten möchte, ist auf ein Menschenbild angewiesen, das grundsätzlich kooperationsbereit ist und unter bestimmten Bedingungen auch in ,unegoistischer“ Weise kooperiert. Unter Zuhilfenahme neuerer Erkenntnisse der Behavioral Economics und in Anlehnung an Elinor Ostroms Konzept der konditionalen Kooperationsbereitschaft haben wir den „bedingten Kooperateur" und seine wesentlichen Annahmen entwickelt und leisten so einen Beitrag zur Debatte um ein zeitgemäßes Menschenbild der Ökonomie.

Darauf aufbauend entwickelten wir einen Bezugsrahmen der Organisation von Kooperation in Unternehmen, um zu zeigen, wie Bedingungen geschaffen werden können, die Kooperationsbereitschaft fördern. Eine besondere Rolle spielen dabei so genannte weiche Integrationsmechanismen wie Reputation, Vertrauen und Solidarität. Nur wenn diese sich herausbilden, agieren Mitarbeiter auch langfristig in unegoistischer Weise kooperativ. Die tatsächliche Kooperation wirkt dann wiederum positiv auf den Aufbau von Reputation, Vertrauen und Solidarität; ein sich selbst verstärkender Zyklus entsteht, durch den sich Kooperationsnormen etablieren. Beeinflusst wird die Entstehung von Kooperationsnormen aber in besonderem Maße auch davon, welche Kooperationspraktiken vorherrschen. Wesentlich ist dabei, dass die Kooperation von den Beteiligten selbst gesteuert wird. So müssen die Beteiligten beispielsweise die Möglichkeit haben, entsprechende Regeln aufzustellen und bei Nichteinhaltung dieser Regeln Sanktionen zu erlassen. Sobald die Akteure erkennen, dass die Kooperation funktioniert, verfestigen sich dann die Kooperationsnormen und fördern so wiederum den Kooperationsprozess.

Insgesamt zeigt der vorliegende Beitrag, dass ein funktionierender Kooperationsprozess von mehreren Faktoren abhängt, die sich in nicht-trivialer Weise gegenseitig beeinflussen. Elinor Ostrom hat demonstriert, was das für langfristige Kooperation in sozio-ökologischen Systemen bedeutet; wir haben ihre Ideen mit organisationstheoretischen Überlegungen verbunden und daraus Schlüsse für die Organisation von Kooperation in Unternehmen abgeleitet. Der Beitrag zur aktuellen Organisationsforschung liegt dabei darin, kooperative Aspekte des menschlichen Handelns in die Diskussion zu integrieren und organisationale Rahmenbedingungen und Steuerungsmöglichkeiten aufzuzeigen.

\section{Literaturverzeichnis}

Adler, P. S. (2002): Corporate Scandals: It's Time for Reflection in Business Schools, in: Academy of Management Executive, Vol. 16/No. 3, 148-149. 
Axelrod, R./Hamilton, W. D. (1981): The Evolution of Cooperation, in: Science, Vol. 211/No. 27, 1390-1396.

Burt, R./Knez, M. (1995): Kinds of Third-Party Effects on Trust, in: Rationality and Society, Vol. 7/No. 3, 255-292.

Camerer, C./Loewenstein, G./Prelec, D. (2005): Neuroeconomics: How Neuroscience Can Inform Economics, in: Journal of Economic Literature, Vol. 43/No. 1, 9-64.

Coch, L./French, J. R. P. (1948): Overcoming Resistance to Change, in: Human Relations, Vol. 1/No. 4, 512-533.

Cox, J. C./Friedman, D./Gjerstad, S. (2007): A Tractable Model of Reciprocity and Fairness, in: Games and Economic Behavior, Vol. 19/No. 1, 17-45.

De Cremer, D./van Dijk, E. (2002): Perceived Criticality and Contributions in Public Good Dilemmas: A Matter of Feeling Responsible to All?, in: Group Processes \& Intergroup Relations, Vol. 5/No. 4, 319-332.

Deutsch, M. (1969): Conflicts: Constructive and Destructive, in: Journal of Social Issues, Vol. 25/No. 1, 7-42.

Deutsch, M. (1975): Equity, Equality, and Need: What Determines Which Value Will be Used as the Basis of Distributive Justice, in: Journal of Social Issues, Vol. 31/No. 3, 137-149.

Elger, C. E. (2008): Neuroleadership: Erkenntnisse der Hirnforschung für die Führung von Mitarbeitern, 1. Aufl., Hamburg: Haufe-Lexware.

Enste, D. H./Haferkamp, A./Fetchenauer, D. (2009): Unterschiede im Denken zwischen Ökonomen und Laien - Erklärungsansätze zur Verbesserung der wirtschaftspolitischen Beratung, in: Perspektiven der Wirtschaftspolitik, Vol. 10/No. 1, 60-78.

Fahr, R./Irlenbusch, B. (2000): Fairness as a Constraint on Trust in Reciprocity: Earned Property Rights in a Reciprocal Exchange Experiment, in: Economic Letters, Vol. 66/No. 3, 275-282.

Falk, A./Febr, E./Fischbacher, U. (2003): On the Nature of Fair Behavior, in: Economic Inquiry, Vol. 41/No. 1, 20-26.

Fehr, E./Gächter, S. (2000): Fairness And Retaliation: The Economics Of Reciprocity, in: Journal of Economic Perspectives, Vol. 14/No. 3, 159-181.

Fehr, E./Gächter, S. (2002): Altruistic Punishment in Humans, in: Nature, Vol. 419/No. 6903, 137-140.

Fehr, E./Schmidt, K. M. (2001): Theories of Fairness and Reciprocity, in: Munich Discussion Paper No. 2001-2, München: Volkswirtschaftliche Fakultät, Ludwig-MaximiliansUniversität, http://epub.ub.uni-muenchen.de/14/1/.

Fischbacher, U./Gächter, S./Fehr, E. (2001): Are People Conditionally Cooperative? Evidence from a Public Goods Experiment, in: Economic Letters, Vol. 71/No. 3, 397-404.

Forde, C./Slater, G./Spencer, D. A. (2006): It's the Taking Part that Counts? Participation, Performance and External Labour Market Conditions, in: Relations Industrielles/Industrial Relations, Vol. 61/No. 2, 296-320.

Franzen, A./Pointner, S. (2008): Fairness und Reziprozität im Diktatorspiel, in: Rehberg, K.-S. (Hrsg.): Die Natur der Gesellschaft: Verhandlungen des 33. Kongresses der Deutschen Gesellschaft für Soziologie in Kassel 2006 (Teilband 1 u. 2), Frankfurt a.M.: Campus Verlag, 2113-2125.

Frey, B. S./Benz, M. (2007): Die psychologischen Grundlagen des Marktmodells (homo oeconomicus), in: von Rosenstiel, L./Frey, D. (Hrsg.): Enzyklopädie der Psychologie. Bd. 5: Marktpsychologie, Göttingen: Hogrefe, 1-26.

Frey, B. S./Humbert, S./Schneider, F. (2010): What is Economics? Attitudes and Views of German Economists, in: Journal of Economic Methodology, Vol. 17/No. 3, 317-332. 
Frey, B. S./Meier, S. (2004): Social Comparisons and Pro-Social Behavior: Testing "Conditional Cooperation" in a Field Experiment, in: American Economic Review, Vol. 94/No. 5, $1717-1722$.

Frey, B. S./Osterloh, M. (2002): Managing Motivation: Wie Sie die neue Motivationsforschung für Ihr Unternehmen nutzen können, 2. Aufl., Wiesbaden: Gabler.

Frost, J./Morner, M. (2005): Corporate Commons: Sustaining Competitiveness Through Public Goods in Multidivisional Firms, in: International Journal of Learning and Change, Vol. 1/No. 1, 28-45.

Frost, J./Morner, M. (2010): Konzernmanagement. Strategien für Mehrwert, 1. Aufl., Wiesbaden: Gabler.

Garfinkel, H. (1963): A Conception of, and Experiments with, Trust as a Condition of Stable Concerted Actions, in: Harvey, O. J. (Ed.): Motivation and Social Interaction: Cognitive Determinants, New York, Ronald, 81-93.

Gouldner, A. W. (1960): The Norm of Reciprocity: A Preliminary Statement, in: American Sociological Review, Vol. 25/No. 2, 161-178.

Gouldner, A. W./Gouldner, H. P. (1963): Modern Sociology - An Introduction to the Study of Human Interaction, Burlingame, New York: Harcourt, Brace \& World.

Granovetter, M. (1985): Economic Action and Social Structure: The Problem of Embeddedness, in: American Journal of Sociology, Vol. 91/No. 3, 481-510.

Kahneman, D. (2003): A Psychological Perspective on Economics, in: American Economic Review, Vol. 92/No. 3, 162-168.

Kahneman, D./Knetsch, J. L./Thaler, R. H. (1991): Anomalies. The Endowment Effect, Loss Aversion and Status Quo Bias, in: The Journal of Economic Perspectives, Vol. 5/No. 1, 193-206.

Kahneman, D./Tversky, A. (1979): Prospect Theory: An Analysis of Decision under Risk, in: Econometrica, Vol. 47/No. 2, 263-291.

Keysar, B./Converse, B.A./Wang, J./Epley, N. (2008): Reciprocity is Not Give and Take - Asymmetric Reciprocity to Positive and Negative Acts, in: Psychological Science, Vol. 19/No.12, 1280-1286.

Kilduff, M./Krackhardt, D. (1994): Bringing the Individual Back In: A Structural Analysis of the Internal Market for Reputation in Organizations, in: Academy of Management Journal, Vol. 37/No. 1, 87-108.

König, E./Volmer, G. (2005): Das Menschenbild der personalen Systemtheorie, in: König, E. (Hrsg.): System und Organisation, Bd. 10, Weinheim, Basel: Beltz Verlag, 33-43.

Kramer, R. M. (1999): Trust and Distrust in Organizations: Emerging Perspectives, Enduring Questions, in: Annual Review of Psychology, Vol. 50, 569-598.

Kirchgässner, G. (2008): Homo Oeconomicus - Das ökonomische Modell individuellen Verhaltens und seine Anwendung in den Wirtschafts- und Sozialwissenschaften, 3. ergänzte und erweiterte Aufl., in: Albert, M. (Hrsg.): Die Einheit der Gesellschaftswissenschaften - Studien in den Grenzbereichen der Wirtschafts- und Sozialwissenschaften, Bd. 74, Tübingen: Mohr Siebeck.

Long, C. P./Bendersky, C./Morrill, C. (2011): Fairness Monitoring: Linking Managerial Controls and Fairness Judgements in Organizations, in: Academy of Management Journal, Vol. 54/No. 5, 1045-1068.

Lubmann, N. (1994): Die Wirtschaft der Gesellschaft, 6. Aufl., Frankfurt a.M.: Suhrkamp.

Mayo, E. (2003/1933): The Human Problems of an Industrial Civilization, in: Thompson, K. (Ed.): The Early Sociology of Management and Organizations, Vol. 6, London, New York: Macmillan. 
McGregor, D. (1966): The Human Side of Enterprise, in: Bennis, W. G./Schein, E. H. (Eds.): Leadership and Motivation, Essays of Douglas McGregor, Cambridge: MIT Press, 3-20.

Meifert, M. T./Kesting, M. (2004): Gesundheitsmanagement - ein unternehmerisches Thema?, in: Meifert, M. T./Kesting, M. (Hrsg.): Gesundheitsmanagement im Unternehmen, Berlin, Heidelberg: Springer-Verlag, 3-13.

Mohr, R. D./Zoghi, C. (2008): High-involvement Work Design and Job Satisfaction, in: Industrial and Labor Relations Review, Vol. 68/No. 3, 275-296.

Morgenstern, O. (1972): Descriptive, Predictive and Normative Theory, in: Kyklos - International Review for Social Sciences, Vol. 25/No. 4, 699-714.

Nowak, M. A. (2006): Five Rules for the Evolution of Cooperation, in: Science, Vol. 314/No. 5805, $1560-1563$.

Osterloh, M./Frey, B. S. (1997): Sanktionen oder Seelenmassage? Motivationale Grundlagen der Unternehmensführung, in: Die Betriebswirtschaft, Vol. 57/No. 3, 307-321.

Osterloh, M./Weibel, A. (2009): The Governance of Explorative Knowledge Production, in: Foss, N. J./Michailova, S. (Eds.): Knowledge Governance - Processes and Perspectives, New York: Oxford University Press, 138-165.

Ostrom, E. (1990): Governing the Commons - The Evolution of Institutions for Collective Action, New York: Cambridge University Press.

Ostrom, E. (1998): A Behavioral Approach to the Rational Choice Theory of Collective Action, in: American Political Science Review, Vol. 92/No. 1, 1-22.

Ostrom, E. (2000): Collective Action and the Evolution of Social Norms, in: Journal of Economic Perspectives, Vol. 14/No. 3, 137-158.

Ostrom, E. (2005): Understanding Institutional Diversity, Princeton, Woodstock: Princeton University Press.

Ostrom, E. (2010a): Analyzing Collective Action, in: Agricultural Economics, Vol. 41/Issue Supplements 1, 155-166.

Ostrom, E. (2010b): Beyond Markets and States: Polycentric Governance of Complex Economic Systems, in: American Economic Review, Vol. 100/No. 3, 641-672.

Owen, H. (2008): Open Space Technology - A User's Guide, 3rd ed., San Francisco: BerrettKoehler Publishers.

Pareto, V. (1969/1906): Manual of Political Economy, New York: Kelley

Posner, R. A./Rasmusen, E. B. (1999): Creating and Enforcing Norms, with Special Reference to Sactions, in: International Review of Law and Economics, Vol. 19/No. 3, 369-382.

Poteete, A. R./Janssen, M. A./Ostrom, E. (2010): Working Together - Collective Action, the Commons, and Multiple Methods in Practice, Princeton, Woodstock: Princeton University Press.

Rafaeli, A. (1985): Quality Circles and Employee Attitudes, in: Personnel Psychology, Vol. 38/No. 3, 603-615.

Ringlstetter, M. (1997): Organisation von Unternehmen und Unternehmensverbindungen: Einführung in die Gestaltung der Organisationsstruktur, München: Oldenbourg Wissenschaftsverlag.

Seabright, P. (1993): Managing Local Commons: Theoretical Issues in Incentive Design, in: Journal of Economic Perspectives, Vol. 7/No. 4, 113-134.

Simon, H. A. (1955): A Behavioral Model of Rational Choice, in: Quarterly Journal of Economics, Vol. 69/No. 1, 99-118.

Smith, A. (1999/1776): The Wealth of Nations, London: Penguin Books.

Spitzer, M./Fischbacher, U./Herrnberger, B./Grön, G./Fehr, E. (2007): The Neural Signature of Social Norm Compliance, in: Neuron, Vol. 56/No. 1, 185-196. 
Strauss, G. (1998): An Overview, in: Heller, F./Pusić, E./Strauss, G./Wilpert, B. (Eds.): Organisational Participation: Myth and Reality, Oxford: Oxford University Press, 8-39.

Tjosvold, D. (2008): The Conflict-Positive Organization: It Depends Upon Us, in: Journal of Organizational Behavior, Vol. 29/No. 1, 19-28.

Toralla, M. S. P./Falzon, P./Morais, A. (2012): Participatory Design in Lean Production: Which Contribution from Employees? For What End?, in: Work, Vol. 41, 2706-2712.

Tsai, W./Ghoshal, S. (1998): Social Capital and Value Creation: The Role of Intrafirm Networks, in: Academy of Management Journal, Vol. 41/No. 4, 464-476.

Tversky, A./Kahneman, D. (1986): Rational Choice and the Framing of Decisions, in: Journal of Business, Vol. 59/No. 4, 251-278.

von Neumann, J./Morgenstern, O. (1947): Theory of Games and Economic Behavior, Princeton: Princeton University Press.

Vroom, V. H. (1964): Work and Motivation, New York: Wiley \& Sons.

Walras, L. (1954/1874): Elements of Pure Economics: Or the Theory of Social Wealth, Cambridge: Harvard University Press.

Wedekind, C. (1998): Game Theory: Give and Ye Shall Be Recognized, in: Science, Vol. 280/No. 5372, 2070-2071.

Willer, R./Fynn, F. J./Zak, S. (2012): Structure, Identity, and Solidarity: A Comparative Field Study of Generalized and Direct Exchange, in: Administrative Science Quarterly, Vol. 57/No. 1, 119-155.

Williamson, O. E. (1985): The Economic Institutions of Capitalism - Firms, Markets, Relational Contracting, New York: The Free Press. 